\title{
Topography of Neutron Scattering from Diblock Copolymer Film
}

\author{
Zhong-hou Cai \\ Argonne National Laboratory, 9700 South Cass Avenue, Argonne, IL 60439
}

T. P. Russell

IBM Research Division, Almaden Research Center, 650 Harry Road, San Joes, Ca 95120

\begin{abstract}
The surface structure of a multilayered film of symmetric perdeuterated diblock copolymers of polystyrene and polymethylmethacrylate, denoted P(d-S-b-dMMA), was investigated by neutron scattering at grazing incidence. The film surfaces were covered by islands or holes of uniform height with a lateral size of several micrometers. With the angle of incidence fixed, the off-specular neutron scattering was measured as function of scattering angle and neutron wavelength. The off-specular scattering intensity shows ridges at constant $\mathrm{q}_{\mathrm{z}}$, the momentum transfer along the surface normal. The scattering arises from the surface domains. It was found that the off-specular ridges developed from minima in the oscillations in the specular profile corresponding to domain height. The characteristic of the off-specular scattering, which is different from that of the off-specular scattering due to the conformal roughness in a multilayered film, is interpreted in terms of the correlations of the surface domains.
\end{abstract}

\section{DISCLAIMER}

This report was prepared as an account of work sponsored by an agency of the United States Government. Neither the United States Government nor any agency thereof, nor any of their employees, makes any warranty, express or implied, or assumes any legal liability or responsibility for the accuracy, completeness, or usefulness of any information, apparatus, product, or process disclosed, or represents that its use would not infringe privately owned rights. Reference herein to any specific commercial product, process, or service by trade name, trademark, manufacturer, or otherwise does not necessarily constitute or imply its endorsement, recommendation, or favoring by the United States Government or any agency thereof. The views and opinions of authors expressed herein do not necessarily state or reflect those of the United States Government or any agency thereof. 


\section{DISCLAIMER}

This report was prepared as an account of work sponsored by an agency of the United States Government. Neither the United States Government nor any agency thereof, nor any of their employees, make any warranty, express or implied, or assumes any legal liability or responsibility for the accuracy, completeness, or usefulness of any information, apparatus, product, or process disclosed, or represents that its use would not infringe privately owned rights. Reference herein to any specific commercial product, process, or service by trade name, trademark, manufacturer, or otherwise does not necessarily constitute or imply its endorsement, recommendation, or favoring by the United States Government or any agency thereof. The views and opinions of authors expressed herein do not necessarily state or reflect those of the United States Government or any agency thereof. 


\section{DISCLAIMER}

Portions of this document may be illegible in electronic image products. Images are produced from the best available original document. 


\section{INTRODUCTION}

Conformal roughness, the roughness or waviness of the individual interfaces within a multilayered film which is fully or partially replicated from one interface to the next, is often found in films consisting of thin layers and has received significant attention recently.1,2 Because of strong correlations of roughness or long-wavelength waviness between interfaces, the off-specular scattering arising from these lateral inhomogeneities exhibits constructive interference. Conformal roughness is usually observed in off-specular scattering along $\mathrm{q}_{\mathrm{z}}$, the momentum transfer of the scattering along the surface normal, where the intensity of diffuse scattering shows a similar $\mathrm{q}_{z}$ dependence as that in specular reflection. This characteristic is called "in phase" between specular and off-specular scattering.

Another interesting surface topography occurs when islands or holes of uniform height form on the surface of a film. In this case, strong off-specular scattering is expected even though all interfaces in the film are ideally smooth. Similar to the case of a multilayer with conformal roughness, the off-specular scattering from such surface features yield coherent scattering along $q_{z}$, which offers information of domain height. However, the lateral correlations of fluctuations for these two cases are much different. This raises the question as to how lateral correlations affect the off-specular scattering?

Thin films of symmetric diblock copolymers offer an ideal system to address this question. Thin films of symmetric diblock copolymers, that is copolymers in which the number of segments in each block are equal, upon annealing form, in most cases, a lamellar structure with the lamellae oriented parallel to the surface of the substrate. ${ }^{3-5}$ This mandates that the film thickness at any point be defined as $(\mathrm{m}+1 / 2) \mathrm{L}$ or $\mathrm{mL}$, where $\mathrm{m}$ is an integer and $\mathrm{L}$ is the period of the lamellar structure. ${ }^{6-9}$ The two different possibilities arise from the preferential affinities of the blocks to the substrate and air interfaces. For films of arbitrary thickness not satisfying these conditions, islands or holes are formed on the surface with a step height equal to the lamellar period. Typically, the lateral size of the islands or holes are on the order of micrometers. 
Herein, an experimental study, using off-specular neutron scattering, of the surface structure of thin films of symmetric diblock copolymers of deuterated polystyrene and polymethylmethacrylate [denoted $\mathrm{P}(\mathrm{d}-\mathrm{S}-\mathrm{b}-\mathrm{d}-\mathrm{MMA})$ ] is presented. It was found that the lateral correlations of the surface topography dominate the offspecular scattering, which can be interpertated by the formalism developed previously. 10

\section{EXPERIMENTAL}

The copolymer used in the studies was purchased from Polymer Laboratories and had a total molecular weight of $100 \mathrm{~K}$. The fraction of PS segments in the copolymer was 0.5 . The copolymer was purified by Soxhlet extraction in cyclohexane to remove any PS homopolymer impurity before use. Films of the copolymer were prepared on 5-cm-diameter polished $\mathrm{Si}$ substrate using standard spin coating methods. Here, $3 \%(\mathrm{w} / \mathrm{v})$ solutions of the copolymer in toluene were filtered, spread onto the Si surface and spun at variable speeds to adjust the thickness of the film. The specimens were heated to $170{ }^{\circ} \mathrm{C}$ under vacuum for 90 hours to order the copolymer and then quenched to a temperature below the glass transition of the copolymer to stabilize the structure. Figure 1 shows a large scale atomic force microscopy (AFM) image of the surface of an annealed P(d-S-b-d-MMA) film in which domains are formed on the surface. A height profile of AFM showed that domain heights are nearly uniform.

Neutron scattering measurements were performaed using the POSY II reflectometer at the Intense Pulsed Neutron Source (IPNS) at Argonne National Laboratory. ${ }^{11}$ The geometry of the experiment is shown in figure 2 . Neutrons of wavelength between 2.5 and $16 \AA$ are selected by two reflection filters from the incident white beam and collimated by two slits $72 \mathrm{~cm}$ apart at an incident angle $\theta_{i}$. In normal operation, the angular resolution of the beam is controlled by the slits so that $\delta \theta_{\mathrm{i}} / \theta_{\mathrm{i}}$ is kept about 0.05 in runs at different incident angles. A linear position sensitive detector with 256 channels ( $175 \mathrm{~cm}$ from the sample) detected the scattered and reflected neutrons. The detector subtended an angle $\theta_{\mathrm{i}}+\theta_{\mathrm{f}}=6^{\circ}$. Each channel is divided into 256 time-of-flight channels. The time resolution is set considerably higher than the angular resolution. Thus, the spectrometer enables one to measure 
$1 \mathrm{~g}$ intensity as a function of scattering angle $\theta_{\mathrm{f}}\left(\right.$ or $\left.\phi=\theta_{\mathrm{i}}+\theta_{\mathrm{f}}\right)$ and the of neutrons.

\section{ULTS}

nents were performed on an annealed thin film of P(d-S-b-d-MMA) on is on the surface. The film was first characterized by $x$-ray reflectivity ular scattering as described previously. 10 By x-ray reflectivity, the film o have a total thickness of $1910 \AA$. Surface domains were observed by microscopy and off-specular x-ray scattering. A domain height of 423 ned from a scattering measured in an offset $\theta-2 \theta$ scan. Because PMMA $y$ resides at the silicon or the $\mathrm{S}_{\mathrm{i}} \mathrm{O}_{2}$ surface, and PS is located at the free total film thickness has the form $(m+1 / 2) \mathrm{L}$. Therefore, the film is if 3.5 full layers of -PMMA-PS-PS-PMMA- lamellar period and an layer of the lamallar period at the surface.

fferential cross section of the scattering from such a film can be rithin the kinematical scattering theory, as ${ }^{10}$

$$
\begin{aligned}
& \sum_{j}^{V} \alpha_{i} \alpha_{j} \sum_{m=i-1}^{i} \sum_{n=j-1}^{j}(-1)^{n+m+i+j} \frac{\rho_{m} \rho_{n}^{*}}{q_{z, m} q_{z, n}^{*}} \exp \left[-i\left(q_{z, m} z_{i}-q_{z, n}^{*} z_{j}\right)\right] \\
& \operatorname{xp}\left[-\frac{\left(q_{z, m}^{2} \sigma_{i}^{2}+q_{z, n}^{* 2} \sigma_{j}^{2}\right)}{2}\right] \iint_{A} d X d Y\left[P_{i j}(X, Y)+1\right] \exp \left[-i\left(q_{x} X+q_{y} Y\right)\right]
\end{aligned}
$$

llid when the incident and the scattered angles are far from the critical al reflection or the angle of incidence is such that it does not satisfy the 'the Bragg reflection (if the film possesses the structure of the Bragg In Eq. (1), $N$ is the number of the layers in the film, $i$ and $j$ refer to ith indices from 1 (air interface) to $N$ (substrate surface), $m$ and $n$ refer im 0 (air) to $N$ (substrate), $A$ is the total area of the sample, $\rho_{m}$ is the ngth density of layer $\mathrm{m}, \mathrm{q}_{\mathrm{z}, \mathrm{m}}$ is the momentum transfer of the scattering $m$ along the surface normal, $\mathrm{q}_{\mathrm{x}}$ and $\mathrm{q}_{\mathrm{y}}$ are the lateral components of the transfer, $z_{i}$ is the average height of the interface $i$, and $\sigma_{i}$ is the root 
mean square roughness of interface $\mathrm{i}$. In $\mathrm{Eq} .(1), \mathrm{P}_{\mathrm{ij}}(\mathrm{X}, \mathrm{Y})$ is the coherent contribution of the total correlation function of domains and roughness between interfaces $i$ and $j$; that is

$$
\begin{aligned}
P_{i j}(X, Y) & =h(X, Y) R_{i j}+h(X, Y)+R_{i j} & & i, j \leq l-1 \\
& =\frac{\alpha^{2} h(X, Y) R_{i j}}{(1-\alpha)^{2}}+\frac{\alpha^{2} h(X, Y)}{(1-\alpha)^{2}}+R_{i j} & & i, j=l \\
& =R_{i j}-\frac{\alpha h(X, Y)}{1-\alpha}-\frac{\alpha h(X, Y) R_{i j}}{1-\alpha} & & i \leq l-1, \mathrm{j}=1 \text { or } \mathrm{i}=1, j \leq l-1 \\
& =R_{i j} & & \text { else, }
\end{aligned}
$$

where the index 1 refers to the interface supporting surface domains, $\alpha$ is the surface coverage of domains, $\alpha_{i}$ is the coverage of the interface i. It is $\alpha$ if the interface contains domains, it is $(1-\alpha)$ if the interface supports domains, and it is 1 if the interface is completed. $h(X, Y)$ and $R_{i j}$ are the coherent parts of the correlation functions of surface domains and interfacial roughness, respectively. 10

In Eq. (1), the summation of $m$ (or $n$ ) from $i-1$ to $i$ (or from $j-1$ to $j$ ) takes account of the difference in scattering length densities of media at both sides of the interface $i$ (or $j$ ). When interface $i$ supports surface domains the ( $i-1$ )-th medium is air instead of the common (i-1)-th layer. It should be noted that the two terms in the integral of Eq. (1) correspond to the contributions of off-specular scattering $\left[\mathrm{P}_{\mathrm{ij}}(\mathrm{X}, \mathrm{Y})\right]$ and specular reflectivity $(1)$, respectively.

Figure 3 shows the topography of neutron scattering from the $P(d-S-b-d-M M A)$ diblock copolymer film. The scattering intensities are plotted versus an angle, $\phi=\theta_{\mathrm{i}}+\theta_{\mathfrak{f}}$, and the neutron wavelength $\lambda$. The incidence angle $\theta_{\mathrm{i}}$ was fixed at $1.6^{\circ}$. At a given neutron wavelength when $\theta_{\mathrm{f}}$ is close to the critical angle of total reflection for that wavelength, an anomalous scattering (or Yoneda scattering) occurs. As the wavelength of neutrons varies (as can be seen from the figure), it forms a scattering band at large wavelength and small scattering angle regions. It is found that the maximum intensities of the band move along a line of constant $k_{f}$ in the $\phi-\lambda$ plane, where $\mathrm{k}_{\mathrm{f}}\left(\mathrm{k}_{\mathrm{f}}=2 \pi \sin \theta_{\mathrm{f}} / \lambda \approx 2 \pi \theta_{\mathrm{f}} / \lambda\right)$ is the momentum of the scattered neutrons along the surface normal. The critical angle for neutrons can be written as 


$$
\theta_{c}=\lambda\left(\frac{b \varsigma}{\pi}\right)^{1 / 2}
$$

where $b$ is the characteristic scattering length, and $S$ is the number density of the scattering particles. Yoneda scattering occurs when the Eq. (3) is satisfied. The scattering forms a straight line in $\phi-\lambda$ plane. It should be noted that Eq. (1) is no longer valid when $\phi$ and $\lambda$ are such that $k_{f}$ approaches $k_{c}$, the critical wave vector of the polymer film. However, in the Distorted Wave Born Approximation (DWBA), Eq. (1) can be corrected by a factor of $\left|\mathrm{T}\left(\theta_{\mathrm{i}}\right)\right|^{2} \mid \mathrm{T}\left(\theta_{\mathrm{f}}\right)^{2}$, where $\mathrm{T}$ is the Fresnel transmission function. 12

Within reflection geometry where $\phi=3.2^{\circ}$, the reflectivity shows oscillations as the neutron wavelength varies. These oscillations are due to the interference of the neutrons reflected by the top and bottom of the film, and, hence, the frequency of the oscillations contains the information of total film thickness. It is also seen that the amplitudes of these oscillations are modulated at a frequency four and one half times lower than that of the primary oscillations. This is due to the presence of holes on the surface and the beating of the frequencies arising from the height (or thickness) differences between the surface and stepped holes in the film.

When the geometry of the scattering is off the specular reflection, for $\phi<3.2^{\circ}$, as the wavelength of the neutrons varies, oscillation are seen with a frequency equal to that of the beat frequency. Previously it was found that there is neither conformality nor partial comformality of the roughness of the interfaces within the films. ${ }^{10}$ Thus, the structured off-specular scattering must arise from surface features. The contrast of scattering length densities at the top surface (i') and at the interface supporting the domains $\left(j^{\prime}\right)$, that is in the holes, is much larger than those of the underlying interfaces of domain. The scattering from the two interfaces will coherently interfere along $\mathrm{q}_{\mathrm{z}}$, which is represented by the first exponential in Eq. (1) for the elements relating to the two interfaces. In other words, the existence of holes or islands on the film surface provides the system with a reciprocal lattice vector perpendicular to the surface. It is well known that whenever $q_{z}$ becomes the reciprocal lattice vector or a higher order of the vector, say $\mathrm{q}_{z}^{\prime}$, strong scattering occurs. The factors $\exp \left[-\mathrm{i}\left(\mathrm{q}_{\mathrm{z}, \mathrm{m}} \mathrm{z}_{\mathrm{i}}^{\prime}-\mathrm{q}^{*}{ }_{\mathrm{z}, \mathrm{n}} \mathrm{z}_{j}\right)\right]$ and $\exp \left[-\mathrm{i}\left(\mathrm{q}_{\mathrm{z}, \mathrm{m}_{\mathrm{m}} \mathrm{z}_{\mathrm{j}}{ }^{\prime}-\mathrm{q}^{*} \mathrm{z}, \mathrm{n}} \mathrm{z}_{\mathrm{i}}{ }^{\prime}\right)\right]$ produce oscillations with a frequency associated with domain height $z_{i}^{\prime}-z_{j}^{\prime}$. 
At constant $\mathrm{q}_{z}^{\prime}$ where a maxium in the scattering occurs, the scattering angle $\phi$ and the wavelength of the neutrons are related by the required momentum transfer of the neutrons, that is

$$
\lambda=\frac{2 \pi}{q_{z}^{\prime}}\left(\sin \theta_{i}+\sin \theta_{f}\right) \approx \frac{2 \pi}{q_{z}^{\prime}} \phi,
$$

where it is assumed that $\theta_{\mathrm{i}}$ and $\theta_{\mathrm{f}}$ are small. As a result, the scattering intensities show the bands. A plot of the maxima of scattering intensities from the topography of Fig. 3 is shown in Fig. 4. As can be seen, straight lines are found nearly passing through the origin in the $\phi-\lambda$ plane. Within these lines, the momentum transfer of neutrons along the surface normal is constant and can be described by Eq. (4). From the slopes of the lines, $\mathrm{q}_{\mathrm{z}}^{\prime}$ can be obtained, which characterizes the reciprocal lattice vector of the system corresponding to the height of domains at the surface. Here only three lines are resolvable corresponding to the $\mathrm{q}_{\mathrm{z}}$ values of 0.0393 , 0.0544 , and $0.0695 \AA^{-1}$. A domain height of $415 \AA$ is deduced from these values of $\mathrm{q}_{\mathrm{z}}^{\prime}$, in excellent agreement with the period of lamellae in the film. Because there is no scattering due to conformal roughness, the bands of scattering arise from a coherent scattering of the surface domains.

Fig. 5 is the same scattering as that in Fig. 3 but with a view angle along the $\phi$ axis. This view clearly demostrates that the bands of scattering originate in the minima of the low frequency beating oscillations in the reflectivity profile. The behavior of this scattering is much different from that due to conformal roughness. As will be discussed in the next section, the characteristic of the off-specular scattering is determined by the correlations of the surface domains.

\section{DISCUSSION}

For a given structure of a multilayered film, as shown in Eq. (1), the behavior of the off-specular scattering is mainly determined by two factors: the $q_{z}$-dependent part corresponding to the film structure along the surface normal; and the $q_{x}$ dependent part relating to the lateral structure of the film. It is also clear that the $\mathrm{q}_{z^{-}}$ dependent part appears in both specular and off-specular terms, and, when mapped as function of $\mathrm{q}_{\mathrm{z}}$, the intensity profiles of both specular and off-specular scattering 
offer structral information normal to the surface. In the specular scattering because all such structural information appears in the reflectivity profile, it is often difficult to resolve the film structure. However, only those features that have both perpendicular and lateral correlations will be observed in the off-specular scattering along $\mathrm{q}_{\mathrm{z}}$. Thus, the off-specular scattering, combined with the specular reflection, can be a very powerful tool in analyzing the structure of a multilayered film.

A multilayered film can be a very complicated system. In terms of layer structure it could be completed or incompleted, and in terms of the interfacial roughness it could be conformal or nonconformal. Consider a film with domains on the surface and a film with conformal interfacial roughness. Both structures relating to domain height (in the first case) and to the layer thickness (in the second case) are involved in lateral correlations. Consequently, both show bands of scattering that have identical frequency oscillations along $q_{z}$ if the domain height and the layer thickness are equal (as is the case here). Thus, it is important to understand how to determine the film structure from specular and off-specular scattering. The manner in which lateral correlations can be obtained from the off-specular scattering will not be treated here because this was discussed previously. 10 Several different cases will be treated below.

(1) For a completed film without interfacial roughness, both the domain correlation function, $h(X, Y)$, and roughness correlation function, $R_{i j}$, are zero. Hence, $P_{i j}(X, Y)=0$. Thus, only the specular component is left in Eq. (1).

(2) For a completed film with nonconformal interfacial roughness, $h(X, Y)=0$ and $R_{i j} \neq 0$ if $i=j$. The off-specular scattering contains only the diagonal elements in the Eq. (1), that is the off-specular scattering is a superposition of the scattering arising from the roughness of individual interfaces and is incoherent along $q_{z}$.

(3) For a completed film with conformal roughness, $P_{i j} \neq 0$ because $R_{i j}=R_{i i} \neq 0$. As a result, beside that described in case (2), the off-specular scattering contains the interference between the scattering due to the roughness of individual interfaces, which is coherent along $q_{z}$, offering the structural information of layer thickness. Moreover, because the Fourier transform of $R_{i j}$ is greater than zero, the intensity of the off-specular scattering exactly follows that of the specular scattering as $q_{z}$ varies. Therefore, the off-specular and specular scattering are in phase. It should be noted 
that if the scattering length density difference between layers is weak, then the coherent scattering is weak and will, more than likely, not be observed. In most cases, the coherent scattering due to conformal roughness can be clearly seen in the off-specular scattering.

(4) For a film with surface domains and without interfacial roughness, $R_{\mathrm{ij}}=0$ and $P_{i j} \neq 0$, because $h(X, Y) \neq 0$. As in the conformal roughness case, the off-specular scattering is also coherent along $\mathrm{q}_{\mathrm{z}}$. This is the origin of the bands in the scattering shown in Fig. 3. A major difference from case (3) is the function $P_{i j}(X, Y)$, where $i$ and $\mathrm{j}$ refer to air-polymer interfaces. For simplicity, we ignore the refraction and absorption of the radiation by the film. The function form of $P_{\mathrm{ij}}(X, Y)$ or $P_{\mathrm{ji}}(X, Y)$ in Eq. (2) is $-\alpha h(X, Y) /(1-\alpha)$. The component of the off-specular scattering due to the interferences from the two surface heights is

$$
\begin{aligned}
& \left(\left.\frac{d \sigma}{d \Omega}\right|_{i j}+\left.\left.\frac{d \sigma}{d \Omega}\right|_{j i}\right|_{o f f-s p e c u l a r}=-\frac{2 A \alpha^{2} \rho^{2}}{q_{z}^{2}} \cos \left[q_{z}\left(z_{i}-z_{j}\right)\right]\right. \\
& \times \iint_{A} d X d Y h(X, Y) \exp \left[-i\left(q_{x} X+q_{y} Y\right)\right],
\end{aligned}
$$

while the same interference in the specular scattering can be expressed by

$$
\left(\left.\frac{d \sigma}{d \Omega}\right|_{i j}+\left.\frac{d \sigma}{d \Omega}\right|_{j i}\right)_{\text {specular }}=\frac{8 \pi^{2} A \alpha(1-\alpha) \rho^{2}}{q_{z}^{2}} \cos \left[q_{z}\left(z_{i}-z_{j}\right)\right] \delta\left(q_{x}\right) \delta\left(q_{y}\right)
$$

Because the integral in Eq. (5) is always larger than zero, the minus sign in the offspecular scattering component shifts the phase of the oscillations of $\cos \left[\mathrm{q}_{\mathrm{z}}\left(\mathrm{z}_{\mathrm{i}}-\mathrm{z}_{\mathrm{j}}\right)\right]$ one half period and becomes exactly out of phase with the corresponding oscillations in the reflectivity profile. Including the interfacial roughness will not alter this observation. Because the scattering length density difference for neutrons at the surface is large, the off-specular scattering due to surface domains is easily seen. In Fig. 6a, a calculated scattering surface is shown using Eq. (1), which has been corrected by the square of the transmission function of $\theta_{f}$, from a model film of $P(d-$ S-b-d-MMA). No convolutions of the instrument resolution function have been included. In this calculation, only the air and substrate interfaces were considered. Each interface had a nonconformal roughness of $5 \AA$. The total thickness of the film 
was $1870 \AA$, and the height of domains was $415 \AA$. There is good agreement in basic features between the calculated and measured scattering surfaces. The bands of scattering can be clearly seen, which (as can be seen in Fig. 6b) develop from the minima of the same oscillations in the reflectivity profile.

(5) For a film with both surface domains and conformal roughness, the offspecular scattering should generally exhibit coherent scattering along $\mathrm{q}_{\mathrm{z}}$. However, its phase relation with the corresponding component in the reflectivity will depend upon the Fourier transformation of $R_{i j}-\alpha h(X, Y) /(1-\alpha)-\alpha h(X, Y) R_{i j} /(1-\alpha)$. If the average size of domains is much larger than the correlation length of the conformal roughness, the scattering due to the domains will be confined to a very small region at $\mathrm{q}_{\mathrm{x}}=0$, while the tail of the scattering will be mainly determined by the conformality of the roughness. Thus, it is possible that in a region of small $\mathrm{q}_{z}$, where $q_{x}$ is small and $h(X, Y)$ is the dominating term, the off-specular scattering appears out of phase. While for large $q_{z}$, where $R_{i j}(X, Y)$ becomes important, the off-specular scattering appears in phase. Between the two regions, there would be a regime where the off-specular scattering would exhibit less pronounced oscillations along $\mathrm{q}_{\mathrm{z}}$.

The behavior of the off-specular scattering is determined by the lateral correlations of the inhomogeneity parallel to the surface. The phase relation between the specular and off-specular scattering characterizes the source of the off-specular scattering and, hence, is a very useful tool in analyzing the structure of multilayered film with lateral inhomogeneities. Time of flight measurements, which utilize a white neutron source, provide a rapid and useful means by which the phase relations can be extracted.

In summary, specular and off-specular neutron scattering from a thin film of symmetric diblock copolymers of P(d-S-b-d-MMA) with domains of uniform height on the surface have been presented. At grazing incidence, any structure with height and lateral correlations exhibits bands of scattering in the off-specular scattering. These bands move along the lines of constant momentum transfer along the surface normal and are coherent. Also, these bands were found to develop from the minima of the oscillations of a beating frequency in specular reflectivity corresponding to the discrete height changes in the film. It was found that the characteristics of the scattering are an intrinsic property of the scattering from surface topography. The 
off-specular scattering is much different from that due to the conformal roughness in a multilayered film. All features of the scattering could be interpreted using the formalism developed previously. ${ }^{10}$

\section{ACKNOWLEDGMENTS}

We would like to thank G. P. Felcher for helpful discussions. This work was supported by the US Department of Energy, BES-Materials Sciences, under contract W-31-109-ENG-38. 


\section{REFERENCES}

1. D. E. Savage, J. Kleiner, N. Schimke, Y. H. Phang, T. Jankowski, J. Jacobs, R. Kariotis, and M. G. Lagally, J. Appl. Phys., 69, 1411 (1990).

2. S. K. Sinha, Physica B173, 25 (1991).

3. G. E. Molau, "Colloidal and Morphological Behavior of Block and Graft Copolymers," in Block Polymers, S. L. Aggarwal, ed., New York: Plenum Press (1970).

4. G. Coulon, T. P. Russell, V. R. Deline, and P. F. Green, Macromolecules, 22, 2581 (1989).

5. S. H. Anastasiadis, T. P. Russell, S. K. Satija, and C. F. Majkrzak, J. Chem. Phys., 92, 5677 (1990).

6. D. Ausserre, D. Chatenay, G. Coulon, and B. Collin, J. Phys. (Paris) 51, 2571 (1990).

7. G. Coulon, D. Ausserre, and T. P. Russell, J. Phys. (Paris) 51, 777 (1990).

8. G. Coulon, B. Collin, D. Ausserre, D. Chatenay, and T. P. Russell, J. Phys. (Paris) 51, 2801 (1990).

9. M. Maaloum, D. Ausserre, D. Chatenay, and G. Coulon, Phys. Rev. Lett., 68, 1575 (1992).

10. Z. H. Cai, K. G. Huang, P. A. Montano, T. P. Russell, J. M. Bai, G. W. Zajac, J. Chem. Phys., 98, 2376 (1993).

11. A. Karim, B. H. Arendt, R. Goyette, Y. Y. Huang, R. Kleb and G. P. Felcher, Physica B173, 17 (1991).

12. S. K. Sinha, E. B. Sirota, S. Garoff, and H. B. Stanley, Phys. Rev., B38, 2297 (1987). 


\section{FIGURE CAPTIONS}

1. (a) AFM image of relief domains at the surface of a diblock copolymer film of P(d-S-b-d-MMA). The height of the holes was found to be 415 $\AA$ from the height profile obtained from AFM.

2. Schematic illustration of the geometry of the neutron scattering experiment. Scattered neutrons are measured by a linear-sensitive detector with 256 position channels. Each position channel is divided into 256 time-of-flight channels. Data are collected during such a time period that the neutrons of wavelength between 2 and $16 \AA$ are measured.

3. The topography of neutron scattering from a PS/PMMA diblock copolymer thin film with relief domains at the surface. The incident angle $\theta_{\mathrm{i}}$ was fixed at $1.6^{\circ}$. Within specular reflection geometry where $\phi=3.2^{\circ}$, the reflectivity shows oscillations as the neutron wavelength varies. These oscillations are due to the interference of the beams reflected by the top and bottom surfaces of the thin film. For scattering geometry in off-specular condition, at a given neutron wavelength when $\theta_{\mathrm{f}}$ is close to the critical angle for total reflection, an anomalous scattering (or Yoneda scattering) occurs. The topography also shows scattering bands.

4. A plot of the maxima of scattering intensities from the topography in Fig. 3. The scattering bands are along lines of constant $\mathrm{q}_{\mathrm{z}}$ and, hence, are coherently scattered from surface domains. A domain height of $415 \AA$ is deduced from the slopes of these lines, in excellent agreement with the period of lamellae in the film.

5. The scattering topography of Fig. 3 with a view along the $\phi$ axis. The amplitudes of the oscillations within specular reflection are modulated by the oscillations of a lower frequency arising from the surface domains. The scattering bands develop from the minima of the oscillations of low frequency in reflectivity profile. Through a theoretical analysis, it has been found that the characteristic of the off-specular scattering is 
determined by the lateral correlations between domain top surface and the interface supporting domains.

6. (a) Calculated topography of neutron scattering from a model film of $\mathrm{P}(\mathrm{d}-\mathrm{S}-\mathrm{b}-\mathrm{d}-\mathrm{MMA})$ with domains on the surface. The calculation, based on Eq. (1), is corrected by the square of a transmission function for scattered neutrons . (b) Same scattering topography as shown in (a) with a view angle along the $\phi$ axis. The scattering bands develop from the minima of the same oscillations in the reflectivity profile. 

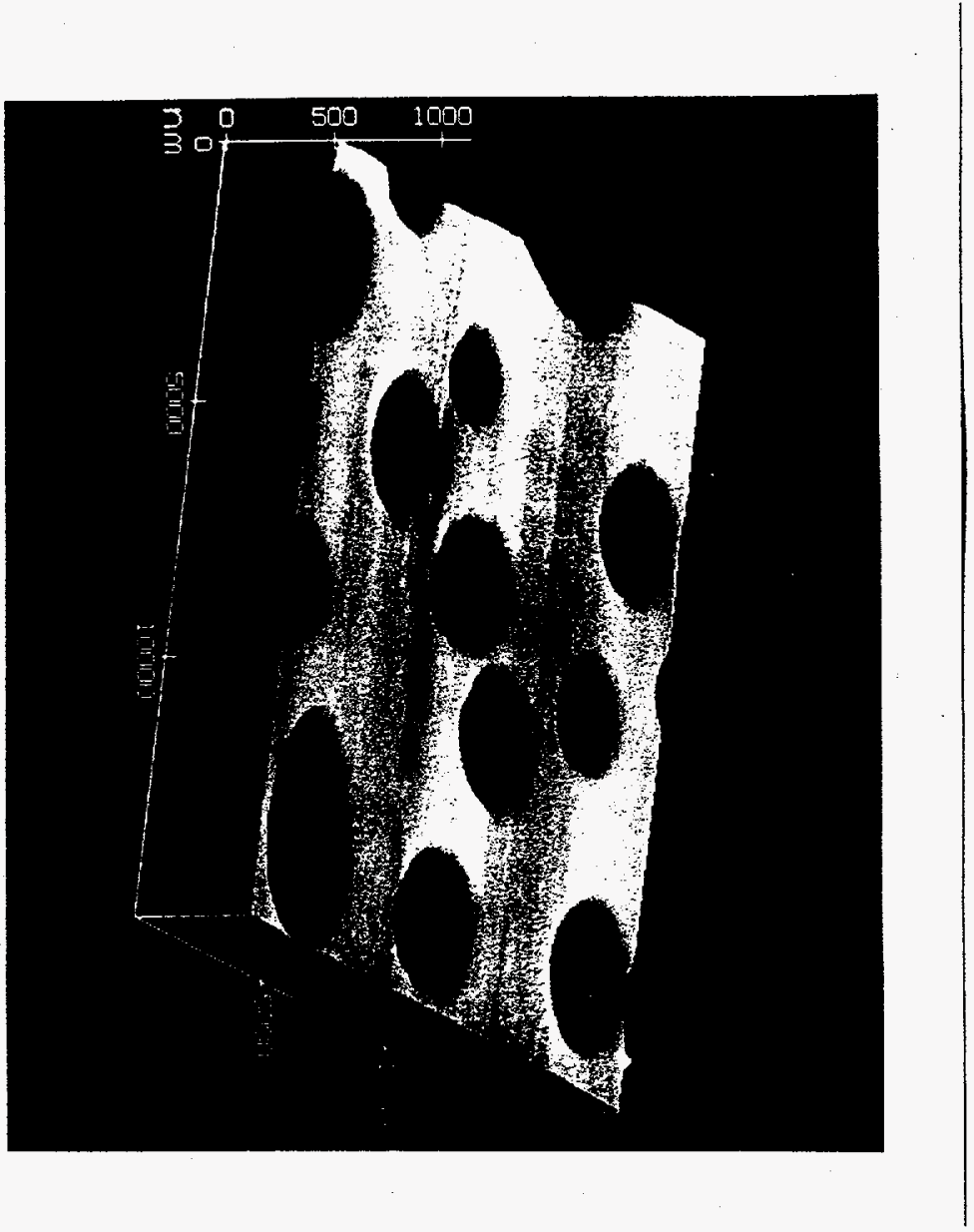


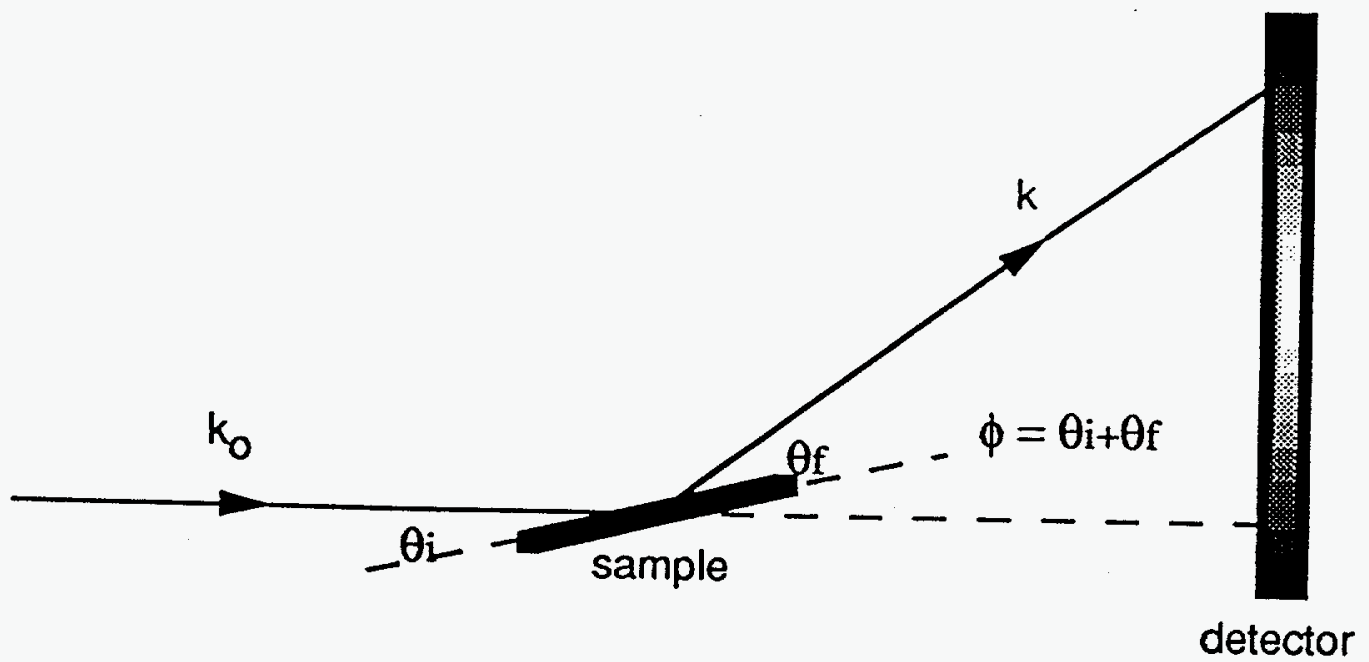




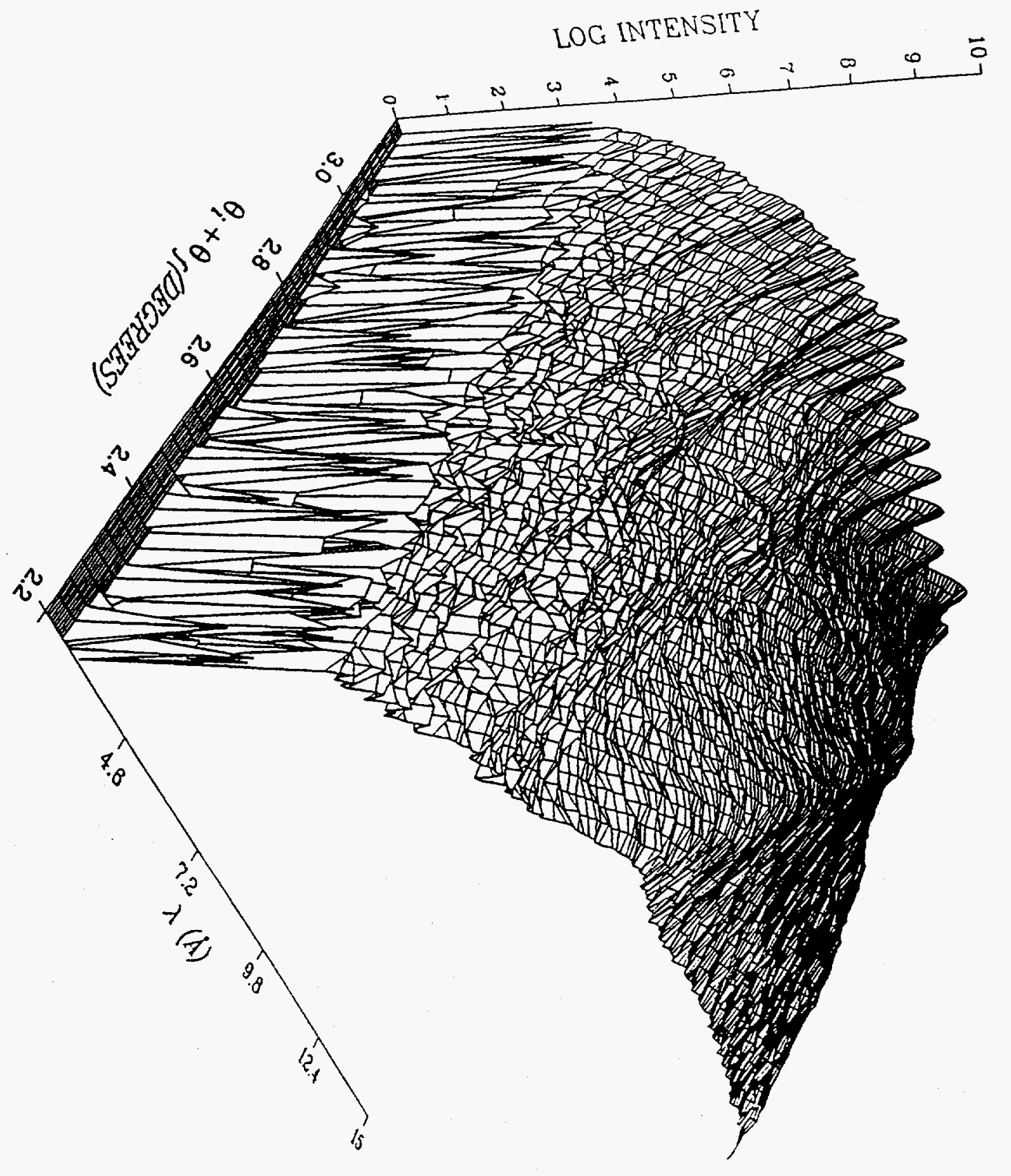




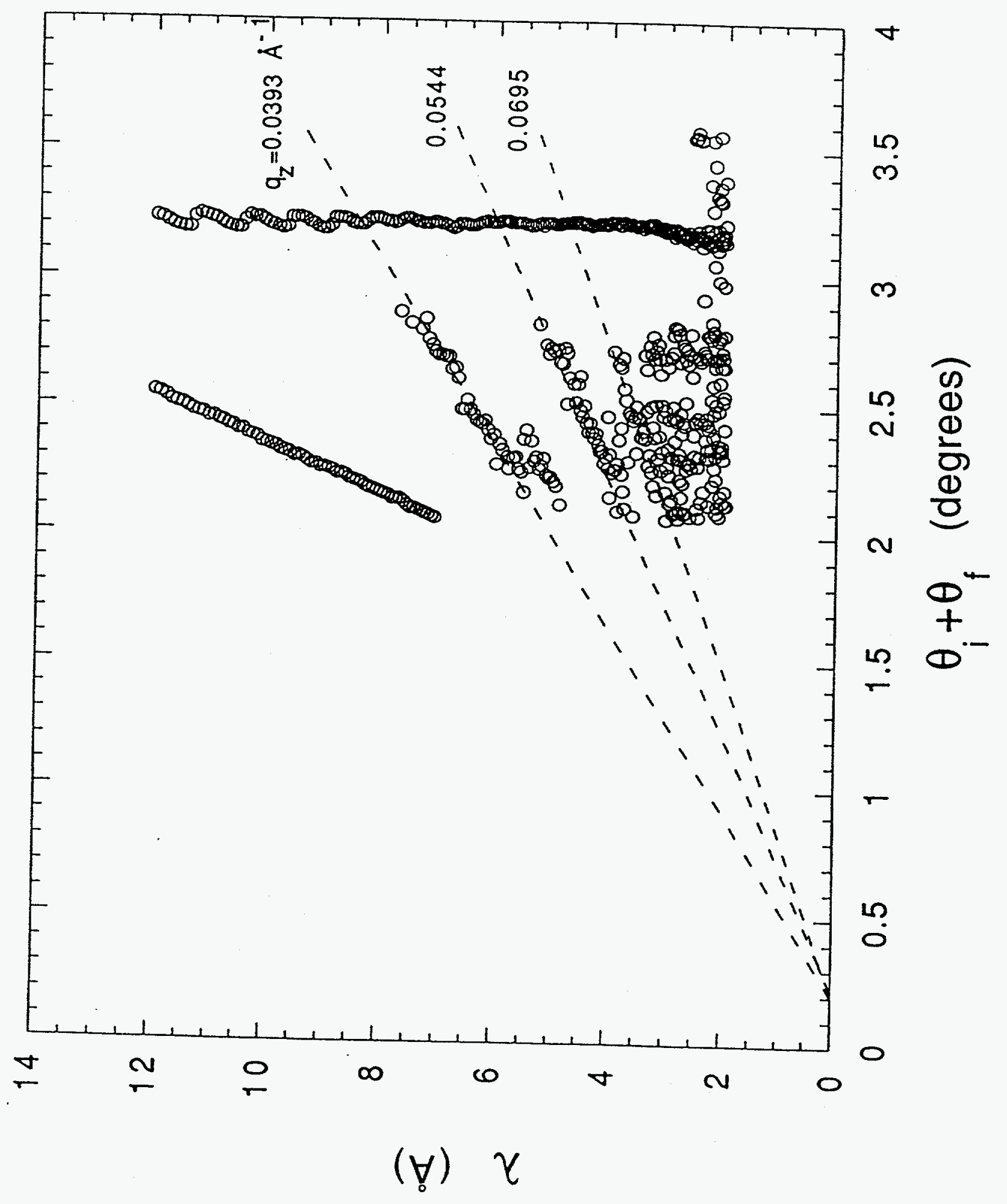




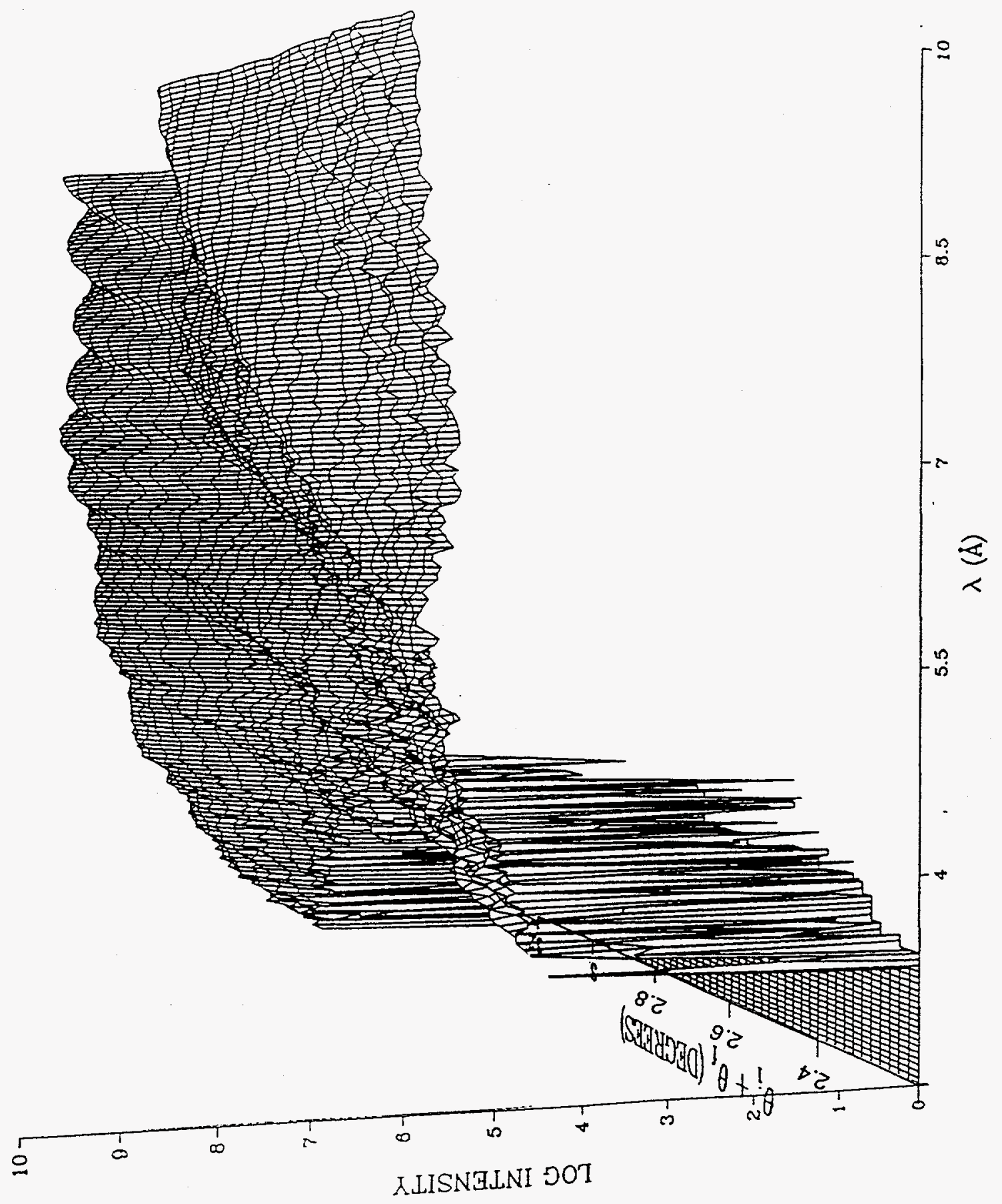




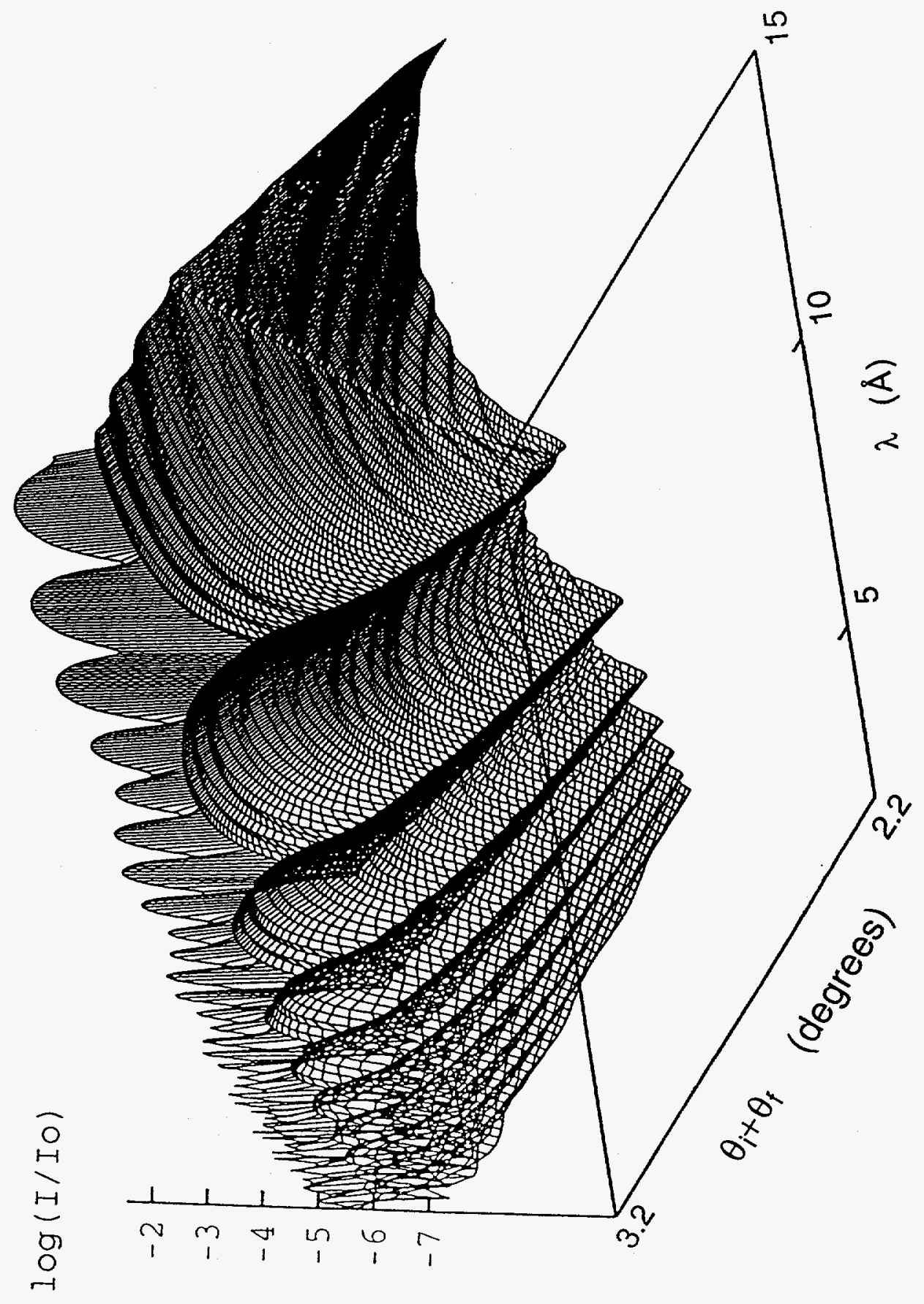




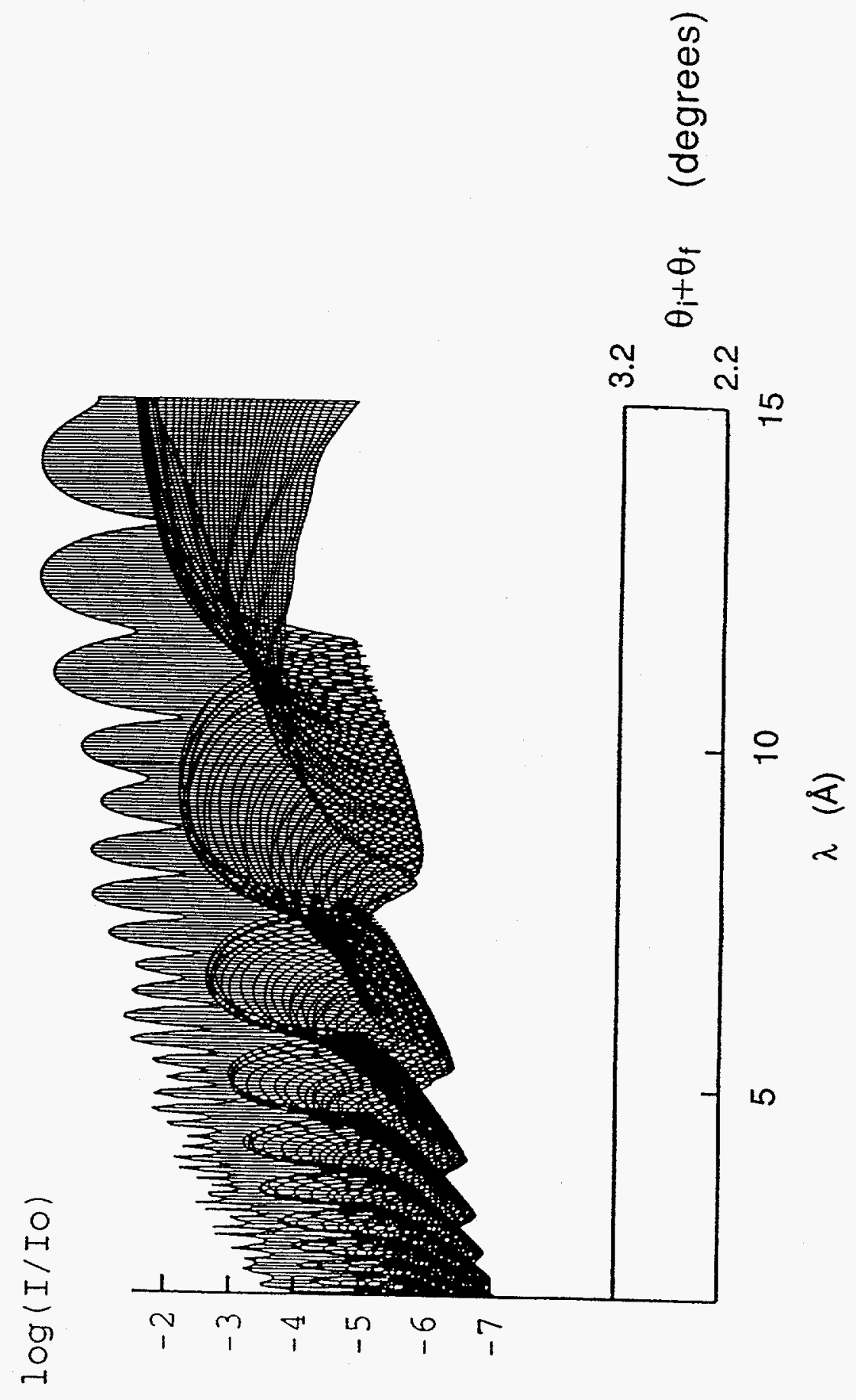

